\title{
THE CHEMISTRY OF THE TRAILING ARM OF THE SAGITTARIUS DWARF GALAXY
}

\author{
Stefan C. Keller, David Yong, and Gary S. Da Costa \\ Research School of Astronomy and Astrophysics, Australian National University, Mt. Stromlo Observatory, Cotter Rd. Weston ACT 2611 Australia; \\ stefan@mso.anu.edu.au \\ Received 2010 April 12; accepted 2010 June 22; published 2010 August 13
}

\begin{abstract}
We present abundances of C, O, Ti, and Fe for $11 \mathrm{M}$-giant stars in the trailing tidal arm of the Sagittarius dwarf (Sgr). The abundances were derived by comparing synthetic spectra with high-resolution infrared spectra obtained with the Phoenix spectrograph on the Gemini South telescope. The targeted stars are drawn from two regions of the Sgr trailing arm separated by $66^{\circ}$ (five stars) and $132^{\circ}$ (six stars) from the main body of Sgr. The trailing arm provides a more direct diagnostic of the chemical evolution of Sgr compared to the extensively phase-mixed leading arm. Within our restricted sample of $\sim 2-3$ Gyr old stars, we find that the stream material exhibits a significant metallicity gradient of $-(2.4 \pm 0.3) \times 10^{-3} \mathrm{dex} /$ degree $\left(-(9.4 \pm 1.1) \times 10^{-4} \mathrm{dex} / \mathrm{kpc}\right)$ away from the main body of Sgr. The tidal disruption of Sgr is a relatively recent event. We therefore interpret the presence of a metallicity gradient in the debris as indicative of a similar gradient in the progenitor. The fact that such a metallicity gradient survived for almost a Hubble time indicates that the efficiency of radial mixing was very low in the Sgr progenitor. No significant gradient is seen to exist in the $[\alpha / \mathrm{Fe}]$ abundance ratio along the trailing arm. Our results may be accounted for by a radial decrease in star formation efficiency and/or radial increase in the efficiency of galactic wind-driven metal loss in the chemical evolution of the Sgr progenitor. The $[\mathrm{Ti} / \mathrm{Fe}]$ and $[\mathrm{O} / \mathrm{Fe}]$ abundance ratios observed within the stream are distinct from those of the Galactic halo. We conclude that the fraction of the intermediate to metal-rich halo population contributed by the recent dissolution ( $<3 \mathrm{Gyr}$ ) of Sgr-like dwarf galaxies cannot be substantial.
\end{abstract}

Key words: galaxies: individual (Sagitarrius dSph) - Galaxy: halo - Galaxy: structure - stars: abundances

Online-only material: color figures

\section{INTRODUCTION}

The paradigm of $\Lambda \mathrm{CDM}$ cosmology enshrines the importance of hierarchical assembly to the process of galaxy formation. Galaxies such as the Milky Way are expected to arise from the coalescence of numerous smaller systems. The idea that the stellar halo of the Milky Way was formed from the disruption of smaller systems was first proposed by Searle \& Zinn (1978). The study of Carollo et al. (2007) argues that the relative contribution of stars originating in external systems to the halo is dependent on galactocentric radius $\left(R_{\mathrm{GC}}\right)$. The outer halo $\left(R_{\mathrm{GC}}>15 \mathrm{kpc}\right)$ is dominated by lower metallicity material and exhibits kinematics indicative of derivation from accretion. On the other hand, at $R_{\mathrm{GC}}<10 \mathrm{kpc}$ the inner halo is dominated by generally higher metallicity and prograde kinematics as would arise from an Eggen et al. (1962) in situ formation scenario. Further, observational studies of the spatial (Bell et al. 2008) and kinematic (Starkenburg et al. 2009) properties of the halo conclude that accretion has a significant role to play in the construction of the halo. These studies find that accretion is responsible for between $10 \%-100 \%$ of the extant population depending on the assumed merger tree for the Galaxy.

An apparent argument against substantial contribution of accreted stars to the halo is the discrepancy between the chemistry of the most-likely accreted systems, namely, dwarf spheroidal (dSphs) galaxies and the chemistry of the halo (see, for example, Unavane et al. 1996, and recently, Tolstoy et al. 2009). At $[\mathrm{Fe} / \mathrm{H}]>-1$ dex, dSph stars exhibit substantially lower $[\alpha / \mathrm{Fe}]$ ratios compared to their Galactic halo counterparts (Tolstoy et al. 2003; Shetrone et al. 2003; Venn et al. 2004). Chemical evolution models (Gilmore \& Wyse 1991; Lanfranchi \& Matteucci 2004) demonstrate that this is due to substantially different star formation histories, combined with differ- ences in the ability to retain processed gas, between the Milky Way and dSph populations. A solution to the chemical mismatch is that the stellar populations of the present-day dSphs are not representative of the bulk of the material previously contributed to the halo via tidal stripping. Indeed, the different chemistry between the present-day $\mathrm{dSphs}$ and the halo might simply reflect that these dSphs were able to survive for a Hubble time and that this has provided an extended timescale for chemical evolution to occur within them (Lagos et al. 2009).

As pointed out by Abadi et al. (2006) and Font et al. (2006), tidal disruption of a dSph in which there is a strong metallicity gradient would lead to the deposition of stars distinct from those surviving in the present-day core. In low mass systems, where orbit swapping processes are expected to be much less effective (Roškar et al. 2008) and distinct from low mass earlytype galaxies that have suffered major mergers (Spolaor et al. 2009), the sense of this metallicity gradient is one of decreasing metallicity with increasing radial distance (Stinson et al. 2009). Furthermore, it has become apparent that the chemistry of some of the most metal-poor $([\mathrm{Fe} / \mathrm{H}]<-3 \mathrm{dex}) \mathrm{dSph}$ stars is indistinguishable from that of the halo (Frebel et al. 2010; Norris et al. 2010). Hence, tidal stripping of the metal-poor outskirts of a dSph could lead to the deposition of material that is old, metal-poor and exhibits abundance ratios matching those of the halo (Cohen \& Huang 2009; Muñoz et al. 2005; Majewski et al. 2003).

In this paper, we examine the chemistry of the stellar population that has been deposited into the outer halo of the Galaxy from the Sagittarius dwarf galaxy (Sgr; Ibata et al. 1994). The tidal disruption of the Sgr dwarf galaxy is the Milky Way's most prominent ongoing accretion event. Tidal debris on leading and trailing arms are traced via RR Lyrae variables (Vivas et al. 
Table 1

Log of Phoenix Observations

\begin{tabular}{|c|c|c|c|c|c|c|c|c|c|c|}
\hline Star & R.A. (J2000) & Decl. (J2000) & $\Lambda_{\odot}($ degrees $)$ & $J$ & $K$ & $\mathrm{E}(B-V)$ & $(J-K)_{0}{ }^{\mathrm{a}}$ & UT Date & Exposure (seconds) & $\mathrm{S} / \mathrm{N}$ \\
\hline $2329301 \mathrm{~m} 245810$ & $23: 29: 30.1$ & $-24: 58: 10$ & 60.00 & 11.590 & 10.499 & 0.02 & 1.145 & 2008-08-21 & $6 \times 562$ & 94 \\
\hline $2345417 \mathrm{~m} 264456$ & $23: 45: 41.7$ & $-26: 44: 56$ & 62.63 & 11.413 & 10.393 & 0.02 & 1.072 & 2008-08-21 & $6 \times 562$ & 98 \\
\hline $2350361 \mathrm{~m} 200216$ & $23: 50: 36.1$ & $-20: 02: 16$ & 66.41 & 11.664 & 10.576 & 0.02 & 1.142 & $2008-09-17$ & $6 \times 562$ & 68 \\
\hline 2353194m205041 & $23: 53: 19.4$ & $-20: 50: 41$ & 66.72 & 12.571 & 11.621 & 0.02 & 1.000 & 2008-09-17 & $12 \times 562$ & 65 \\
\hline $0003528 \mathrm{~m} 194047$ & 00:03:52.8 & $-19: 40: 47$ & 69.35 & 12.024 & 11.007 & 0.03 & 1.069 & 2008-09-19 & $6 \times 562$ & 52 \\
\hline 0334210p051809 & $03: 34: 21.0$ & $+05: 18: 09$ & 126.93 & 12.505 & 11.345 & 0.26 & 1.355 & 2008-09-19 & $12 \times 562$ & 110 \\
\hline 0340164p090338 & $03: 40: 16.4$ & $+09: 03: 38$ & 130.03 & 12.971 & 11.757 & 0.35 & 1.462 & 2009-01-11 & $12 \times 562$ & 30 \\
\hline $0342225 \mathrm{p} 054745$ & $03: 42: 22.5$ & $+05: 47: 45$ & 128.93 & 11.976 & 10.819 & 0.22 & 1.328 & 2008-08-22 & $6 \times 562$ & 78 \\
\hline $0348437 \mathrm{p} 065236$ & $03: 48: 43.7$ & $+06: 52: 36$ & 130.97 & 11.976 & 10.836 & 0.20 & 1.299 & 2009-02-02 & $6 \times 562$ & 97 \\
\hline $0357262 \mathrm{p} 053258$ & $03: 57: 26.3$ & $+05: 32: 48$ & 132.12 & 11.375 & 10.130 & 0.29 & 1.459 & 2008-12-19 & $6 \times 562$ & 79 \\
\hline $0408285 \mathrm{p} 044043$ & 04:08:28.5 & $+04: 40: 04$ & 134.19 & 12.681 & 11.457 & 0.40 & 1.501 & 2008-09-19 & $6 \times 562$ & 65 \\
\hline
\end{tabular}

Notes. The first five stars correspond to the " 66 degree" group, and the remaining six correspond to the " 132 degree" group discussed in the text.

${ }^{a}$ Dereddened color in the Johnson-Glass system.

2004; Keller et al. 2008; Prior et al. 2009), blue horizontal branch stars (Yanny et al. 2000, 2009; Newberg et al. 2007), M giants (Yanny et al. 2009; Majewski et al. 2003), subgiants (Keller et al. 2009), and main-sequence turn-off stars (Belokurov et al. 2006; Jurić et al. 2008). The tidal debris extends around the sky and represents multiple orbits of Sgr around the Milky Way.

This study utilizes high-resolution IR spectroscopy obtained on the Gemini South telescope to target stars in two regions along the length of the Sgr trailing arm. This enables us to determine the chemistry of material lost on two consecutive perigalactic passages of Sgr and hence to investigate the presence of any chemical abundance gradients evident in the stripped material.

\section{SAMPLE SELECTION}

The targets for our abundance study are taken from the M giants cataloged in Majewski et al. (2003). We selected two samples of stars seen toward the trailing arm of Sgr debris that possess radial velocities and distances appropriate for the trailing arm. The radial velocities of the target sample are shown in Figure 1. The two samples at $\Lambda_{\odot}=66^{\circ}$ and $132^{\circ}$ are shown in Figure 1 in the Sgr plane in the context of literature models for the Sgr debris (Law et al. 2005; Fellhauer et al. 2006; Helmi \& White 2001). As seen in the simulations of Law et al. (2005), the two regions represent stars lost from the Sgr system approximately $0.5 \mathrm{Gyr}$ and $1.3 \mathrm{Gyr}$ ago, respectively.

We have chosen to focus on the trailing arm for the following reasons. Firstly, Law et al. (2005) show that the leading arm debris is much less spatially differentiated with respect to the epoch at which material was stripped compared with the trailing arm material (see their Figure 1). Consequently, stars lost in successive orbits overlap significantly. Furthermore, the radial velocity distribution of leading arm stars is less coherent than that seen in the trailing arm (see Law et al. 2005, Figure 10). For trailing arm material selection on the basis of radial velocity is more stringent, and contamination from field star interlopers is less likely than that for a corresponding leading arm sample.

\section{SPECTROSCOPIC OBSERVATIONS}

Table 1 reports the details of our observations. The spectra of individual stars along the trailing arm of Sgr were obtained in the $H$ band at a resolution of $R=50000$. The spectra were obtained using the $8 \mathrm{~m}$ Gemini South telescope and the Phoenix spectrograph (Hinkle et al. 2003) in queue mode observing (Program GS-2008B-Q-33). The observations were
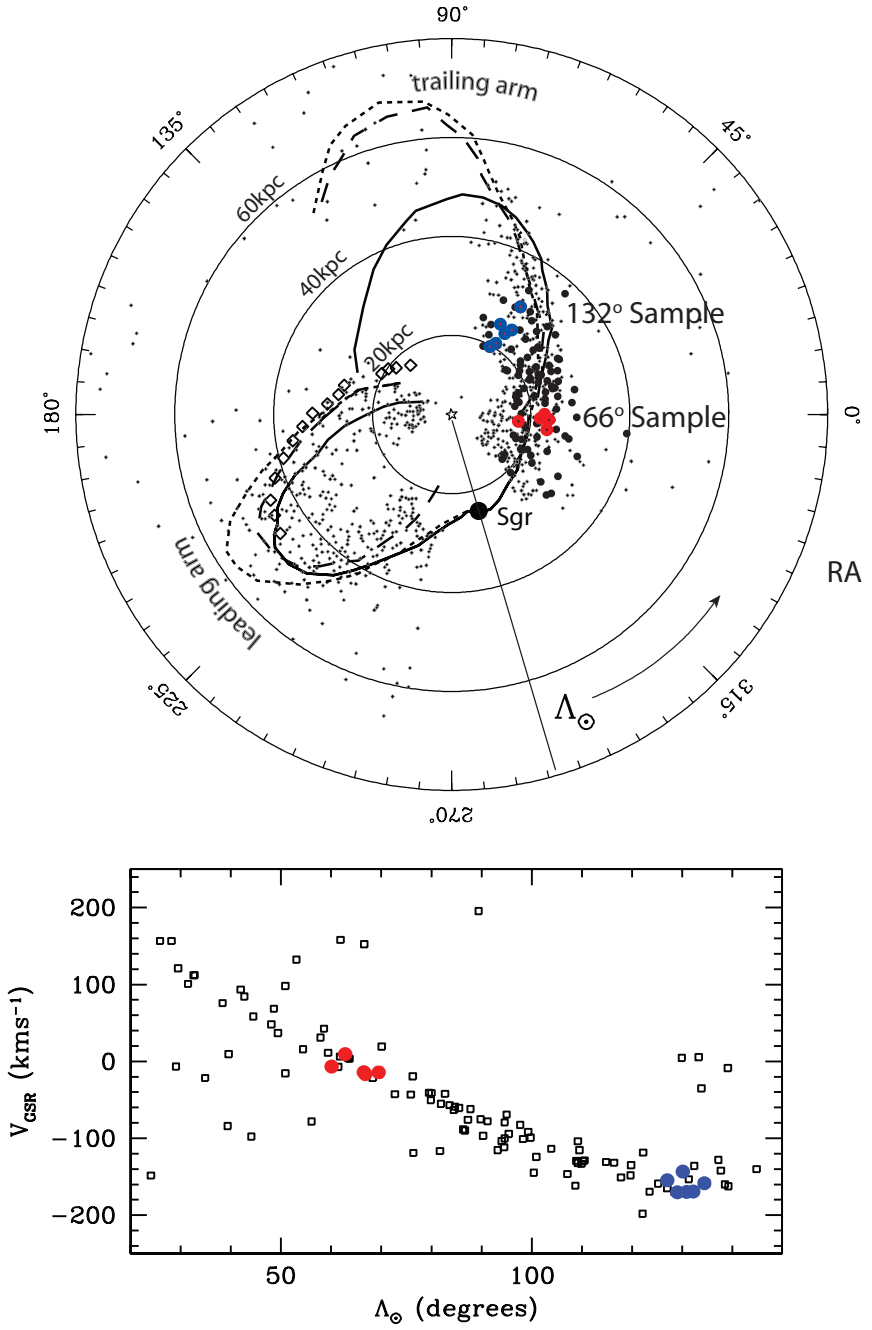

Figure 1. Top: spatial distribution of target stars in the debris stream of Sgr (red: $\Lambda=66^{\circ}$ and blue: $\Lambda=132^{\circ}$ ) projected in R.A. Concentric circles show heliocentric distances of 20-80 kpc. M giants from Majewski et al. (2003) (bold black: radial velocities present and appropriate for Sgr material) and detections from Belokurov et al. (2006) (boxes) are shown. Overlaid are models from Law et al. (2005, solid line), Fellhauer et al. (2006, dashed), and Helmi \& White (2001, short dashed). Bottom: the galactocentric radial velocities of the selected targets. Radial velocities are as determined by Majewski et al. (2003).

(A color version of this figure is available in the online journal.)

centered at $1.555 \mu \mathrm{m}$ and utilized the H6420 order separating filter to provide a wavelength coverage of $75 \AA$. The spectra 


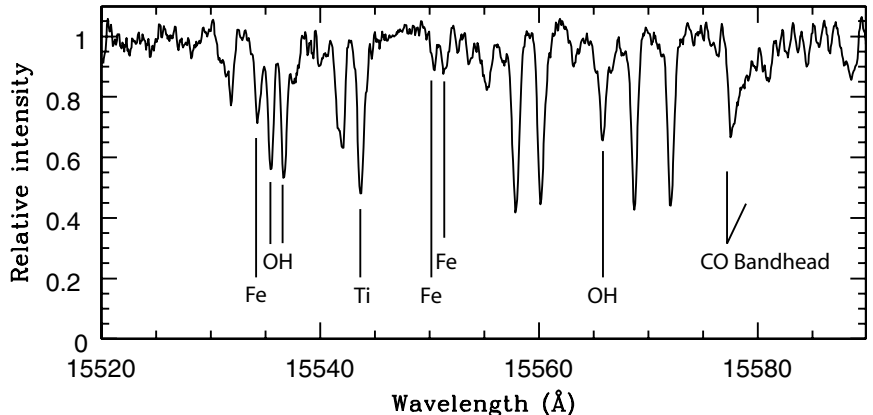

Figure 2. Observed spectrum of $2350361 \mathrm{~m} 200216$. Lines used in the abundance analysis are indicated.

were observed using a 4 pixel slit ( 0.34 wide and $14^{\prime \prime}$ long). Each target star was observed at two positions, left and right of the midpoint of the spectrograph slit length, separated by 2 .. 5 . The sky and dark background were removed by subtracting exposures taken at the alternating positions on the detector array. For each night, 10 dark and flat frames were also acquired. A series of spectra of hot stars for telluric line correction were also acquired. Examination of the observed wavelength window reveals only a very few weak telluric features.

The frames were reduced in the IRAF environment following a procedure similar to that described in Smith et al. (2002) and Meléndez et al. (2003). Dark and flat frames are combined and the resulting dark is subtracted from the flat. A response image was derived from the flat and the science frames were then divided through by this response frame. The spectra were then extracted and wavelength calibrated using the stellar absorption lines evident. Finally, the spectra corresponding to left and right displacements were combined and continuum normalized.

\section{STELLAR PARAMETERS AND ABUNDANCE DETERMINATION}

The reddening to each object is derived from the $100 \mu \mathrm{m}$ Galactic map of Schlegel et al. (1998) and is provided in Table 1. Infra-red photometry is derived from the Two Micron All Sky Survey (2MASS) database having first transformed from the 2MASS system to the Johnson-Glass system ${ }^{1}$ for the purpose of temperature determination. We dereddened the colors of the target stars using a ratio of $E(J-K) / E(B-V)=$ 0.53 (Bessell et al. 1998). The effective temperature scale for cool giants is well defined from occultation measurements. We use the relation of Bessell et al. (1983), namely $\mathrm{T}_{\text {eff }}=$ $7070 /(J-K+0.88)$, to derive the temperatures given in Table 2. Surface gravities were derived from interpolation of appropriate low metallicity isochrones (Marigo et al. 2008). The microturbulent velocity was determined using the following relation, $\xi_{t}=4.2-\left(6 \times 10^{-4} T_{\text {eff }}\right)$, adopted from the optical analysis by Meléndez et al. (2008) of thick disk and bulge stars. We estimate that internal uncertainties in the stellar parameters are $T_{\text {eff }} \pm 75 K, \log g \pm 0.4 \mathrm{dex}$, and $\xi_{t} \pm 0.4 \mathrm{~km} \mathrm{~s}^{-1}$.

Abundances for a given line were derived by comparing synthetic spectra with the observed spectra following the analysis of Yong et al. (2008). The synthetic spectra were generated using the local thermodynamic equilibrium (LTE) stellar line analysis program MOOG (Sneden 1973, 2007 version) and LTE model atmospheres from the MARCS grid (Gustafsson et al. 2008). The line list used in the generation of synthetic spectra was

\footnotetext{
1 http://www.astro.caltech.edu/ jmc/2mass/v3/transformations/
}

Table 2

Derived Stellar Parameters for Target Stars

\begin{tabular}{cccc}
\hline \hline Star & $T_{\text {eff }}(\mathrm{K})$ & $\log g$ & $\xi_{t}\left(\mathrm{~km} \mathrm{~s}^{-1}\right)$ \\
\hline $2329301 \mathrm{~m} 245810$ & 3531 & 0.03 & 2.08 \\
$2345417 \mathrm{~m} 264456$ & 3665 & 0.22 & 2.01 \\
$2350361 \mathrm{~m} 200216$ & 3536 & 0.09 & 2.08 \\
$2353194 \mathrm{~m} 205041$ & 3807 & 1.16 & 1.92 \\
$0003528 \mathrm{~m} 194047$ & 3670 & 0.22 & 2.00 \\
$0334210 \mathrm{p} 051809$ & 3654 & 0.22 & 2.00 \\
$0340164 \mathrm{p} 090338$ & 3646 & 0.16 & 2.01 \\
$0342225 \mathrm{p} 054745$ & 3616 & 0.11 & 2.03 \\
$0348437 \mathrm{p} 065236$ & 3627 & 0.13 & 2.02 \\
$0357262 \mathrm{p} 053258$ & 3526 & 0.02 & 2.08 \\
$0408285 \mathrm{p} 044043$ & 3683 & 0.23 & 1.99 \\
\hline
\end{tabular}

Table 3

Derived Stellar Abundances for Target Stars

\begin{tabular}{ccccc}
\hline \hline Star & $A(\mathrm{C})$ & $A(\mathrm{O})$ & $A(\mathrm{Ti})$ & $A(\mathrm{Fe})$ \\
\hline $2329301 \mathrm{~m} 245810$ & 7.27 & 8.03 & 4.02 & 6.97 \\
$2345417 \mathrm{~m} 264456$ & 7.19 & 7.93 & 4.14 & 6.77 \\
$2350361 \mathrm{~m} 200216$ & 7.34 & 8.13 & 4.29 & 7.02 \\
$2353194 \mathrm{~m} 205041$ & 7.31 & 8.03 & 4.30 & 6.93 \\
$0003528 \mathrm{~m} 194047$ & 7.01 & 7.95 & 4.49 & 6.87 \\
$0334210 \mathrm{p} 051809$ & 7.21 & 7.93 & 4.79 & 7.00 \\
$0340164 \mathrm{p} 090338$ & 7.21 & 7.93 & 4.29 & 6.92 \\
$0342225 \mathrm{p} 054745$ & 6.91 & 8.05 & 4.09 & 6.82 \\
$0348437 \mathrm{p} 065236$ & 6.96 & 7.79 & 3.99 & 6.72 \\
$0357262 \mathrm{p} 053258$ & 7.24 & 8.03 & 4.09 & 6.52 \\
$0408285 \mathrm{p} 044043$ & 7.09 & 7.84 & 3.81 & 6.62
\end{tabular}

Notes. $A(\mathrm{X})=\log [n(\mathrm{X}) / n(\mathrm{H})]+12$. For reference, the Asplund et al. (2006) Solar values of the above elemental abundances are: $A(\mathrm{C})=8.39, A(\mathrm{O})=8.66$, $A(\mathrm{Ti})=4.90$, and $A(\mathrm{Fe})=7.45$.

taken from Meléndez \& Barbuy (1999) and Meléndez et al. (2001, 2003). First, we derived abundances for $\mathrm{O}$ from the $\mathrm{OH}$ molecular lines at $15535.462,15536.705$, and $15565.880 \AA$ (see Figure 2). Next, abundances for $\mathrm{C}$ were obtained from the $\mathrm{CO}$ molecular lines near $15576 \AA$. Since the abundances of $\mathrm{C}$ and $\mathrm{O}$ are coupled, we iterated until self-consistent abundances were obtained, which always occurred within one iteration. $\mathrm{N}$ measurements are possible from $\mathrm{CN}$ lines in the $H$ band. However, examination of the spectra revealed that reliable $\mathrm{N}$ abundances cannot be obtained given the weakness of the $\mathrm{CN}$ lines and the modest signal-to-noise ratios $(\mathrm{S} / \mathrm{N})$. Fe abundances were obtained from the Fe I lines at $15534.260 \AA, 15550.450 \AA$, and $15551.430 \AA$. Ti abundances were determined from the Ti I line at $15543.780 \AA$. In Figure 3 , we show examples of synthetic spectra fits to derive abundances in our sample, and in Table 3 we present the final abundances. In the discussion to follow, we express our abundances relative to the solar values of Asplund et al. (2006). The abundance dependences on the stellar parameters are given in Table 4.

\section{METALLICITY GRADIENT}

In Figure 4, we present our $[\mathrm{Fe} / \mathrm{H}]$ determinations for the two sample regions in the upper panel of Figure 4. In the lower panel of Figure 4, we graphically represent the median $[\mathrm{Fe} / \mathrm{H}]$ and its interquartile range as a function of angular distance $\left(\Lambda_{\odot}\right.$ as defined in Law et al. 2005) from the main body of Sgr. A two-sided Kolmogorov-Smirnov (K-S) test is used to determine the probability that the stream samples were drawn from the core sample (Monaco et al. 2005). The percentages 

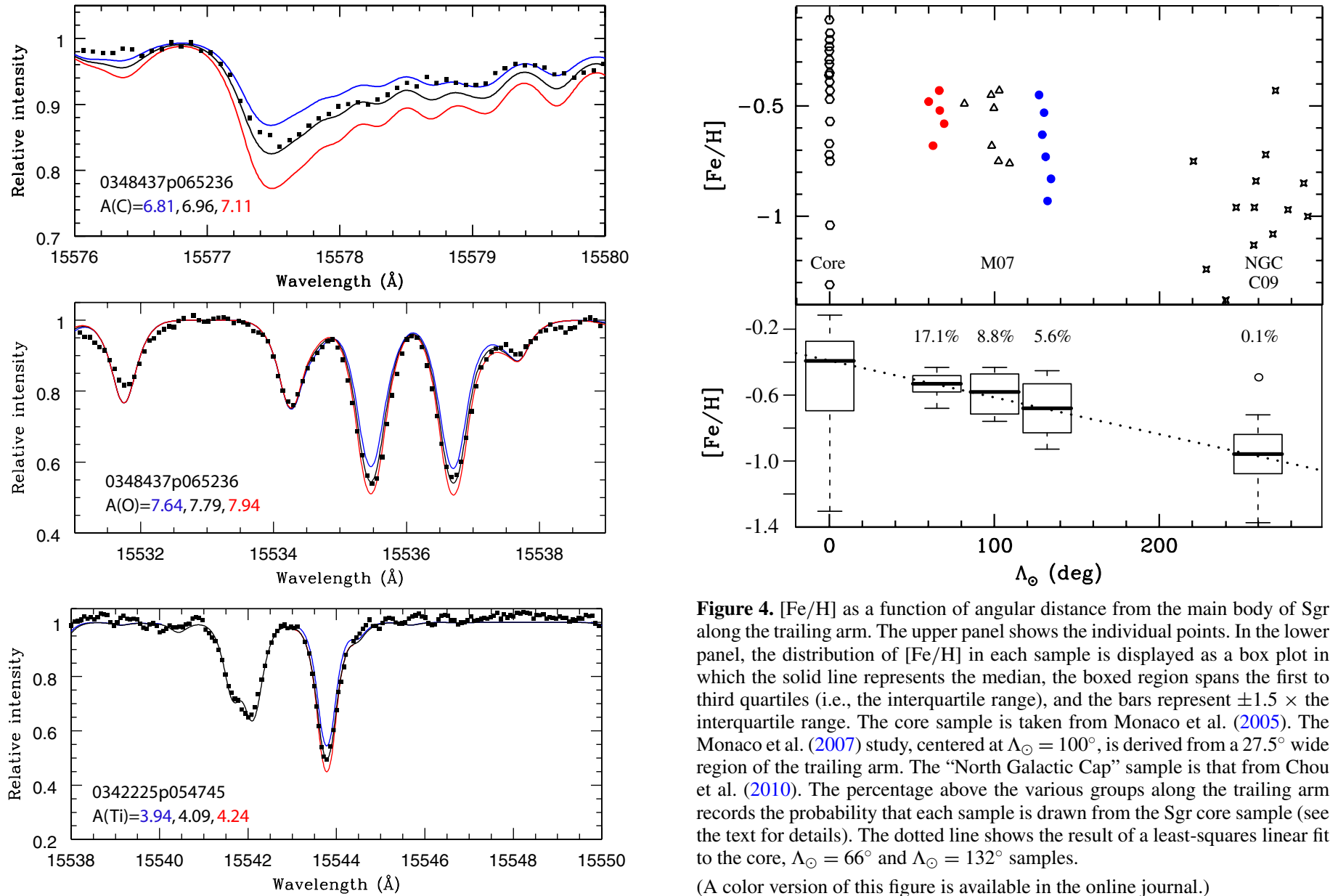

Figure 4. $[\mathrm{Fe} / \mathrm{H}]$ as a function of angular distance from the main body of Sgr along the trailing arm. The upper panel shows the individual points. In the lower panel, the distribution of $[\mathrm{Fe} / \mathrm{H}]$ in each sample is displayed as a box plot in which the solid line represents the median, the boxed region spans the first to third quartiles (i.e., the interquartile range), and the bars represent $\pm 1.5 \times$ the interquartile range. The core sample is taken from Monaco et al. (2005). The Monaco et al. (2007) study, centered at $\Lambda_{\odot}=100^{\circ}$, is derived from a $27.5^{\circ}$ wide region of the trailing arm. The "North Galactic Cap" sample is that from Chou et al. (2010). The percentage above the various groups along the trailing arm records the probability that each sample is drawn from the Sgr core sample (see the text for details). The dotted line shows the result of a least-squares linear fit to the core, $\Lambda_{\odot}=66^{\circ}$ and $\Lambda_{\odot}=132^{\circ}$ samples.

(A color version of this figure is available in the online journal.)

Figure 3. Observed spectra (squares) and synthetic spectra for $\mathrm{C}$ (top), $\mathrm{O}$ (middle), and $\mathrm{Ti}$ (bottom). The synthetic spectra show the best fit (black line) and unsatisfactory fits (red and blue lines), $A(\mathrm{C}, \mathrm{O}, \mathrm{Ti}) \pm 0.15 \mathrm{dex}$.

(A color version of this figure is available in the online journal.)

Table 4

Stellar Abundance Dependences on Model Parameters for 0348437p065236

\begin{tabular}{ccccc}
\hline \hline Species & $T_{\text {eff }} \pm 75 \mathrm{~K}$ & $\log g \pm 0.4 \mathrm{dex}$ & $\xi_{t} \pm 0.4 \mathrm{~km} \mathrm{~s}^{-1}$ & Total $^{\mathrm{a}}$ \\
\hline$\Delta A(\mathrm{C}) \ldots$ & 0.08 & 0.09 & 0.06 & 0.13 \\
$\Delta A(\mathrm{O}) \ldots$ & 0.12 & 0.10 & 0.06 & 0.15 \\
$\Delta A(\mathrm{Ti}) \ldots$ & 0.08 & 0.08 & 0.09 & 0.15 \\
$\Delta A(\mathrm{Fe}) \ldots$ & -0.08 & 0.04 & -0.04 & 0.10 \\
\hline
\end{tabular}

Note. ${ }^{a}$ The total value is the sum in quadrature of the individual abundance dependences.

above each sample in the lower panel of Figure 4 show the probability that this is the case. Low values of this probability indicate that the assumption that the stream samples are similar to that of the core is a poor one. More distant material is seen to be progressively less like the core sample. A gradient of $[\mathrm{Fe} / \mathrm{H}]=-(2.4 \pm 0.3) \times 10^{-3} \mathrm{dex} /$ degree is determined from a least-squares fit to the core, $\Lambda_{\odot}=66^{\circ}$ and $\Lambda_{\odot}=132^{\circ}$ samples. At a mean distance of $22 \mathrm{kpc}$ this projects to $-(9.4 \pm 1.1) \times$ $10^{-4} \mathrm{dex} / \mathrm{kpc}$.

The target stars have been taken from the study of Majewski et al. (2003) and are selected therein on the basis of their 2MASS colors as M-giant stars. The judicious selection of Majewski et al. isolates the upper red giant branch (RGB) of Sgr with low contamination from the Milky Way (MW) field. However, it also imposes a bias toward metal-rich stars as detailed in Majewski

et al. (2003). Metallicities of $[\mathrm{Fe} / \mathrm{H}]<-1$ dex are essentially excluded due to this color selection. To minimize the effects of this imposed metallicity bias on our findings, the above figures compare our results with literature data that impose identical color selection of the $\mathrm{M}$ giants. Utilization of $\mathrm{M}$ giants also imposes an age range to the sample of stars we study. An M giant of $[\mathrm{Fe} / \mathrm{H}]=-0.4 \mathrm{dex}$ (typical of the Sgr core; Monaco et al. 2005 ) possesses an age of 2-2.5 Gyr. At lower metallicities, an older age is required to reach the same $J-K$ color (for example a $1 M_{\odot}[\mathrm{Fe} / \mathrm{H}]=-0.4$ star is 0.1 dex younger than a $[\mathrm{Fe} / \mathrm{H}]=$ -0.7 star at $J-K=1.0$; Marigo et al. 2008).

Our results may be compared to the metallicity gradient observed in the more extensively studied leading arm material. As noted above, the leading arm is more extensively phased mixed. That is to say, the material lost in successive orbits is not as spatially differentiated as in the trailing arm. This effect would be expected to reduce the apparent metallicity gradient along the leading arm compared to the trailing arm. In their study of the leading arm M giants, Chou et al. (2007) report the metallicity distribution function (MDF) in two regions; one centered at $\Lambda_{\odot} \sim 230^{\circ}$ of around $100^{\circ}$ in extent and another region at $\Lambda_{\odot} \sim 30^{\circ}$ (proposed to be old leading arm debris displaced $\sim 390^{\circ}$ from the main body). The mean metallicities are found to be -0.7 dex and -1.1 dex, respectively. Taken together with the mean metallicity of the core, this equates to a metallicity gradient of $-2.2 \times 10^{-3} \mathrm{dex} /$ degree. This is compatible with the present results for the trailing arm.

The $[\mathrm{Fe} / \mathrm{H}]$ gradient we derive here is also compatible with the mean metallicity determined, again from $\mathrm{M}$ giants, in the 
sample of Monaco et al. (2007) (marked M07 in Figure 4). Further, it is noteworthy that our observed $[\mathrm{Fe} / \mathrm{H}]$ gradient continues to the Chou et al. (2007) "North Galactic Cap positive velocity" group which is ascribed by Chou et al. to an old wrap of the trailing arm. The North Galactic Cap sample is not used in our determination of the metallicity gradient of the trailing arm since there is possible confusion with other kinematically distinct substructures in the direction of the North Galactic Cap sample. The North Galactic Cap sample occupies an area of the sky in which there is both leading and trailing arm material as well as material from the Virgo Stellar Stream (Duffau et al. 2006; Vivas et al. 2008; Keller 2010; Prior et al. 2009). The dynamical models of Law et al. (2005) predict that while the leading arm material at the position of the North Galactic Cap sample will possess negative velocities, the trailing material will possess velocities of $100<V_{\mathrm{GSR}}<200 \mathrm{~km} \mathrm{~s}^{-1}$. The Virgo Stellar Stream material possesses a colder velocity profile centered at $V_{\mathrm{GSR}} \sim 100 \mathrm{~km} \mathrm{~s}^{-1}$. Prior et al. (2009) show that considerable overlap in radial velocity exists between the two systems and consequently it is not clear how much of the North Galactic Cap sample is due to the Virgo Stellar Stream. Prior et al. (2009) find that the metallicity of the RR Lyrae members of the Virgo Stellar Stream are uniformly metal-poor at $[\mathrm{Fe} / \mathrm{H}] \sim-1.7$. At such low metallicity, few objects would be contributed to the 2MASS color selection discussed above. However, the RR Lyraes of the Virgo Stellar Stream represent the old stellar population and nothing is known of the metallicity distribution of other populations within this halo substructure.

\subsection{Implications of the Metallicity Gradient in Sgr Debris}

We now discuss the implications of our findings of a metallicity gradient in the stars along the trailing arm of Sgr in the context of models of the chemodynamic evolution of Sgr. Dynamical models of the tidal disruption of Sgr have been presented by numerous authors. Ibata et al. (2001), Helmi (2004), MartínezDelgado et al. (2004), and Law et al. (2005) present models that seek to account for the distance to, and radial velocity of, the M giants of Majewski et al. (2003). Fellhauer et al. (2006) and Martínez-Delgado et al. (2007) include further observational constraints from the Sloan Digital Sky Survey regarding the distance to the Sgr leading arm. A common feature of these studies is that while they manage to qualitatively match the features of the stream, they do not provide a consistent model for the shape of the Milky Way's dark matter halo. In particular, the leading material is best matched by an oblate halo, whereas the trailing material is best matched by a prolate figure (Prior et al. 2009; Newberg et al. 2007; Yanny et al. 2009; Law et al. 2005). Law et al. (2009) demonstrate that by adopting a triaxial halo model, rather than the axisymmetric models assumed in the studies above, a concordant solution is achievable. However, as Law et al. (2009) point out, the solution is somewhat unsatisfactory since such a configuration is expected to be dynamically unstable.

While the above uncertainties remain in the modeling of the orbit of Sgr, the pertinent features for the present study are that stars are released preferentially during perigalactic passage with an orbital period of $\sim 0.85$ Gyr (Law et al. 2005). Consequently, our sample at $\Lambda_{\odot}=66^{\circ}$ was lost from Sgr on the present perigalactic passage approximately $0.5 \mathrm{Gyr}$ ago, and the $\Lambda_{\odot}=$ $132^{\circ}$ sample was lost $\sim 1.3 \mathrm{Gyr}$ ago on the previous passage of Sgr (see Figure 1 of Law et al. 2005).

Studies of the star formation history of the main body of Sgr (Layden \& Sarajedini 2000; Siegel et al. 2007) have revealed a complex and protracted star formation history with three major phases. These studies find an old (11 Gyr) metal-poor population of $[\mathrm{Fe} / \mathrm{H}] \sim-1.3 \mathrm{dex}$, a dominant intermediate age population (6-4.5 Gyr, with possible bursts) with $[\mathrm{Fe} / \mathrm{H}] \sim$ $-0.6 \mathrm{dex}$, and a young (2-3 Gyr) population of $[\mathrm{Fe} / \mathrm{H}] \sim$ -0.4 to $-0.1 \mathrm{dex}$. If the age-metallicity relation (AMR) of $\mathrm{Sgr}$ was spatially uniform then we would expect our samples of $M$ giants to possess the metallicity appropriate for their relatively young age $(\sim 2-3 \mathrm{Gyr})$, namely $-0.4<[\mathrm{Fe} / \mathrm{H}]<-0.1 \mathrm{dex}$ (as dominates the core sample). Furthermore, we would expect a negligible metallicity gradient in the debris stream.

Our observations imply that the progenitor of the present-day Sgr did not possess a spatially uniform AMR. Seen another way, the time between perigalacticons does not allow sufficient time for chemically homogeneous in situ elevation of the mean $[\mathrm{Fe} / \mathrm{H}]$ to the levels we observe between successive orbits. Rather, as recognized by Chou et al. (2007), the abundance gradient observed must arise due to the stripping of the outer regions of the Sgr progenitor over which a metallicity gradient (and/or concomitant age gradient) was present.

Indeed, such population gradients are typically observed in dwarf galaxies. As reviewed by Stinson et al. (2009), dwarf irregular galaxies universally show extended envelopes dominated by old RGB stars. Such halos of old-intermediate age stars are seen across a range of galaxy luminosity and under a range of tidal conditions. Stellar population gradients are notably less distinct among the local dSph galaxies. However, gradients in the morphology of the horizontal branch are not uncommon and are indicative of metallicity and/or age gradients (Harbeck et al. 2001).

It is plausible, therefore, that the original Sgr progenitor possessed an extended and correspondingly metallicity segregated, halo consistent with our observations. As Sgr experienced strong tidal interaction with the Milky Way, the tidal radius of Sgr decreased with each successive orbit, leading to the loss of stars from outer regions of the progenitor. In this picture, it is the intermediate age $M$ stars (having arisen in perhaps the most recent star formation episode) of this outer Sgr halo population that we see in the tidally disrupted debris tails. The radial metallicity gradient imprinted by previous chemodynamical evolution gives rise to the metallicity gradient we observe here. Since the stars of the present study are among the most recent to form in Sgr, the fact that they exhibit a $[\mathrm{Fe} / \mathrm{H}]$ gradient requires that the abundance gradient is long-lived, having survived for approximately a Hubble time. Moreover, our findings imply that radial mixing of enriched material must be of little significance.

\section{ABUNDANCE RATIO GRADIENTS}

In Figures 5 and 6, we show our findings for [Ti/Fe] and $[\mathrm{O} / \mathrm{Fe}]$ as a function of $[\mathrm{Fe} / \mathrm{H}]$. Both figures show a characteristic underabundance of $\mathrm{O}$ and $\mathrm{Ti}$ at given $[\mathrm{Fe} / \mathrm{H}]$ relative to the stars of the Galactic disk and halo. We recall that the $\mathrm{M}$ giants of the present study represent stars of 2-3 Gyr; Galactic disk stars of this age reside on the Galactic locus (the solid line in Figures 5 and 6) at approximately Solar metallicity.

Figure 7 shows the interquartile ranges of the offset in $[\mathrm{O}, \mathrm{Ti} / \mathrm{Fe}]$ at given $[\mathrm{Fe} / \mathrm{H}]$ between our observed sample and that defined in the Galactic disk and halo ${ }^{2}$ as a function of angular distance from Sgr. The probability that each sample is drawn from the core sample is determined by a two-sided K-S test. In the case of both $[\mathrm{O} / \mathrm{Fe}]$ and $[\mathrm{Ti} / \mathrm{Fe}]$, there is no significant

\footnotetext{
2 In the sense $[X / \mathrm{Fe}]_{\mathrm{Sgr}}-[X / \mathrm{Fe}]_{\text {Galactic }}$, where $X$ is the abundance of $\mathrm{O}$ or Ti.
} 


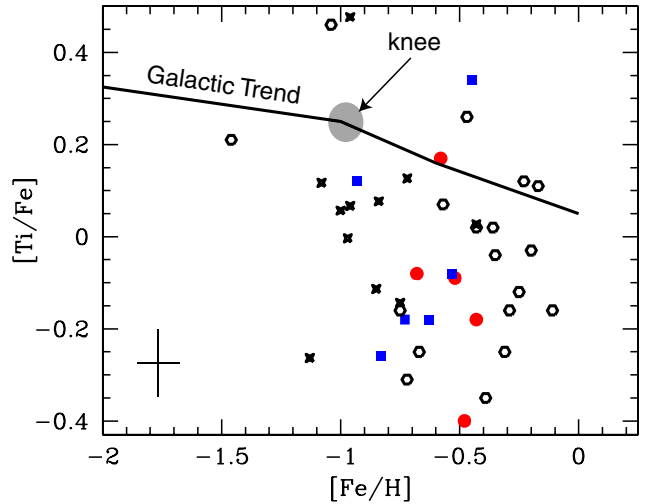

Figure 5. $[\mathrm{Ti} / \mathrm{Fe}]$ vs. $[\mathrm{Fe} / \mathrm{H}]$. Sgr core (open circles; Monaco et al. 2005), trailing arm sample at $\Lambda_{\odot}=66^{\circ}$ (red circles), trailing arm sample at $\Lambda_{\odot}=132^{\circ}$ (blue squares), and North Galactic Cap sample (crosses; Chou et al. (2010)) are shown. For reference the Galactic locus is shown (solid line) as described in Venn et al. (2004). A representative error bar is shown.

(A color version of this figure is available in the online journal.)

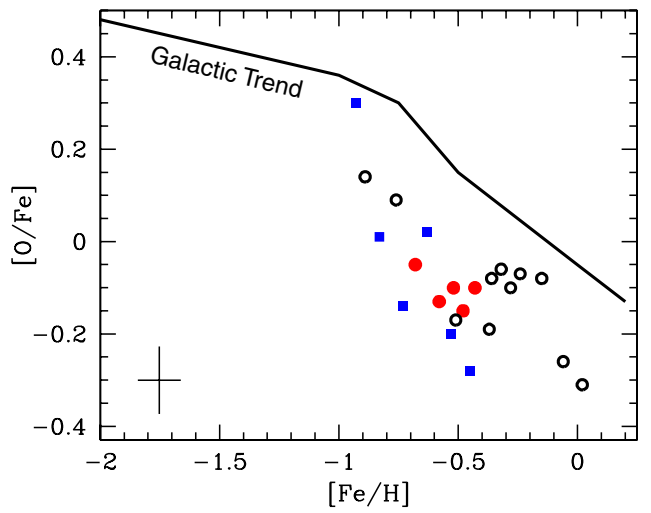

Figure 6. $[\mathrm{O} / \mathrm{Fe}]$ vs. $[\mathrm{Fe} / \mathrm{H}]$. Sgr core (open circles; Sbordone et al. 2007) trailing arm sample at $\Lambda_{\odot}=66^{\circ}$ (red circles), and trailing arm sample at $\Lambda_{\odot}=$ $132^{\circ}$ (blue squares) are shown. For reference the Galactic locus is shown (solid line) as described in Venn et al. (2004). A representative error bar is shown.

(A color version of this figure is available in the online journal.)

gradient along the trailing arm. However, a significant spread in $[\mathrm{Ti} / \mathrm{Fe}]$ is apparent in the four samples in Figure 5. The spread is much larger than the abundance ratio errors (the associated abundance ratio errors of the studies of Monaco et al. (2005) and Chou et al. (2010) are similar in magnitude to those of the present study). Such a spread is not apparent in $[\mathrm{O} / \mathrm{Fe}]$, shown in Figure 6 . The origin of this spread in $[\mathrm{Ti} / \mathrm{Fe}]$ is not understood. Our study does not, however, enable us to define the $[\mathrm{Fe} / \mathrm{H}]$ of the $[\mathrm{O} / \mathrm{Fe}]$ or $[\mathrm{Ti} / \mathrm{Fe}]$ "knee." This is due to the inherent metal-rich bias in our use of $\mathrm{M}$ giants (as discussed above) and small sample size. Consequently, we do not constrain the expected metal-poor $([\mathrm{Fe} / \mathrm{H}]<-1 \mathrm{dex})[\mathrm{O} / \mathrm{Fe}]$ plateau. Chemical evolution models of Lanfranchi \& Matteucci (2004) show that the progressively lower SF efficiency should lead to a $[\alpha / \mathrm{Fe}]$ plateau at progressively lower $[\alpha / \mathrm{Fe}]$ at increasing $\Lambda_{\odot}$. Abundances of radial-velocity selected $\mathrm{K}$ giants (representative of the metal-poor population) in the trailing arm would enable clarification of the location of the "knee" in the $[\alpha / \mathrm{Fe}]$ relation, or otherwise, of a chemical gradient in the trailing arm material.

\subsection{Implications of $\alpha$-element Abundance}

Our observations of the $\alpha$-element $\mathrm{O}$ and the $\alpha$-like element Ti show that the $[\alpha / \mathrm{Fe}]$ ratio of Sgr material is lower at a given $[\mathrm{Fe} / \mathrm{H}]$ compared with that of the MW (at least for

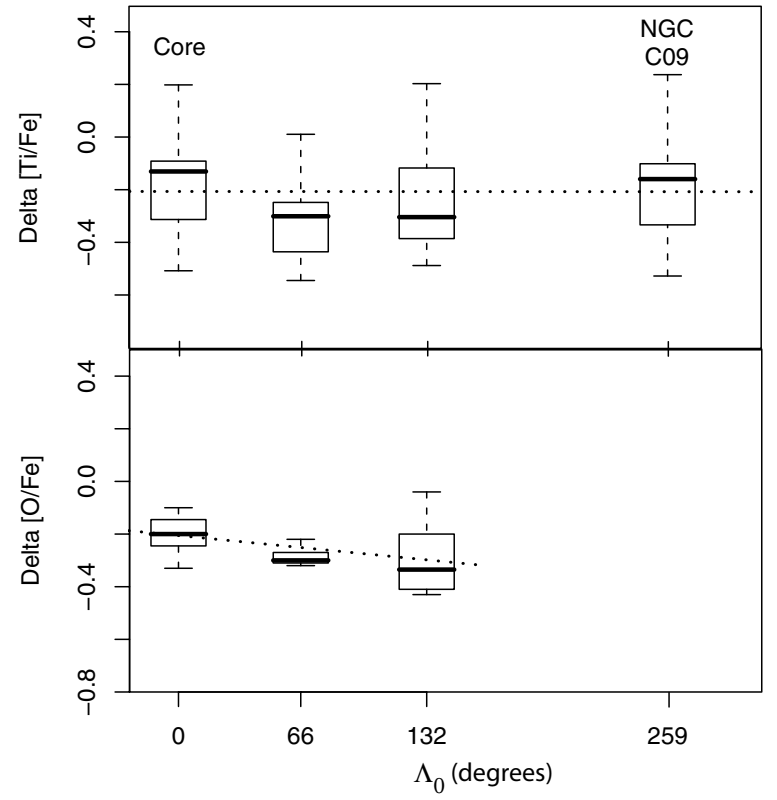

Figure 7. Top: the offset in $[\mathrm{Ti} / \mathrm{Fe}]$ at a given $[\mathrm{Fe} / \mathrm{H}]$ relative to the Galactic halo+disk locus (Venn et al. 2004) as a function of angular distance from the main body of Sgr. The dotted line shows the least-squares linear fit to the samples. Other details of the figure are as described in Figure 4. No spatial gradient in $[\mathrm{Ti} / \mathrm{Fe}]$ is apparent, however, the spread of $[\mathrm{Ti} / \mathrm{Fe}]$ at given $[\mathrm{Fe} / \mathrm{H}]$ is large. Bottom: same as above for $[\mathrm{O} / \mathrm{Fe}]$.

$[\mathrm{Fe} / \mathrm{H}]>-1$ dex material probed to date). As described in a large literature of chemical evolution models (see, for example, Gilmore \& Wyse 1991; Lanfranchi \& Matteucci 2004; Lanfranchi et al. 2006) and motivated by numerous observational studies (for example, Smith et al. 2002; Venn et al. 2004; Cohen \& Huang 2009; Chou et al. 2010; Hidalgo et al. 2009; Lee et al. 2009), the generally lower $[\alpha / \mathrm{Fe}]$ is a consequence of slower chemical enrichment in lower mass systems. The slower pace of chemical enrichment (due to lower star formation efficiency and/or stronger galactic winds) allows Fe-rich SNIa products to be incorporated at lower metallicities relative to the MW halo.

The $[\mathrm{Fe} / \mathrm{H}]$ of the $[\alpha / \mathrm{Fe}]$ "knee" feature, seen in the MW halo at a $[\mathrm{Fe} / \mathrm{H}] \sim-1.0$ dex, provides a snapshot of the metallicity at $\sim 1$ Gyr, after which time SNIa begins to appreciably raise the $\mathrm{Fe}$ content and reduce $[\alpha / \mathrm{Fe}]$. As suggested by Monaco et al. (2005) and demonstrated by Chou et al. (2010) in the Sgr leading arm, for $[\alpha / \mathrm{Fe}] \leqslant-1$ dex there is a general overlap in the $[\alpha / \mathrm{Fe}]$ ratio of MW and Sgr stars. This indicates a common dominance of SNII enrichment at these metallicities. For $[\mathrm{Fe} / \mathrm{H}] \geqslant-1$ dex, however, the $[\alpha / \mathrm{Fe}]$ ratio of Sgr material is significantly below that of the MW. This possibly reflects a lower early star formation rate (SFR) in Sgr compared to the MW, or as proposed by Lanfranchi et al. (2006), that Sgr exhibited a moderate star formation (SF) efficiency at first, but one that was quenched by galactic winds from ensuing SNII.

The implications of the evolution of $[\alpha / \mathrm{Fe}]$ in $\mathrm{Sgr}$ can be contrasted with those in the LMC. In the LMC, the [Ti/Fe] and $[\mathrm{O} / \mathrm{Fe}]$ ratios remain lower than the $\mathrm{MW}$ at all metallicities probed to date $([\mathrm{Fe} / \mathrm{H}] \lesssim-1.3$ (Hill et al. 2000; Smith et al. 2002). This is ascribed to an SF efficiency lower than that of Sgr and/or more efficient galactic winds (discussed in the context of [O/Fe] by Gilmore \& Wyse 1991 \& Smith et al. 2002) and the presence of fewer contributing high-mass SNII (from [Ti/Fe], Pompéia et al. 2008). 
Monaco et al. (2005) therefore point out that the early chemical evolution of Sgr was more akin to that of the MW than that of the LMC and the local dSphs. They propose that the progenitor of Sgr was most likely a gas-rich, star forming galaxy that was substantially more massive than the extant remnant. The Lanfranchi et al. (2006) results amplify this argument. The study of Lanfranchi et al. defines a sequence of relative SFR in early galaxy evolution that ranges from the $\mathrm{dSphs}$ which exhibit the lowest SFR to the LMC with a moderate SFR and finally Sgr with the highest SFR evident among the MW satellites. Thus, the advanced chemical evolution of Sgr material argues strongly that the progenitor was more massive than the LMC. However, at present the dynamical models of Law et al. (2005) assume a progenitor mass of $2-5 \times 10^{8} M_{\odot}$ compared to the LMC mass of $1-2 \times 10^{9} M_{\odot}$. An increase in the progenitor mass of Sgr would imply a more extended history of mass-loss to the MW and an enhanced impact on the chemistry of the halo. We also note that a large mass progenitor would be in line with the observed extended SFH of Sgr that indicates that Sgr was actively forming stars until 1-2 Gyr ago (Siegel et al. 2007; indeed if it had not, there would be no $\mathrm{M}$ giants with which to trace the tidal streams) although it has been tidally interacting with the MW for $\sim 3$ Gyr (see, for example, Law et al. 2005). The ability to retain the gas required for such star formation in the face of Galactic tidal interaction would argue for a large progenitor mass.

\subsection{Sgr and the Formation of the Galactic Halo}

An important role is expected for accreted satellites in the formation of the Galactic halo within $\Lambda$-CDM cosmology. Here, hierarchical mergers lead to massive galaxies surrounded by a flotilla of lower mass satellite halos (at least as traced by dark matter-only simulations). Hence, the stellar content of the halo must presumably contain some contribution from the disruption of such satellites. A number of observational challenges to such a scenario have arisen.

Firstly, the number of lower mass halos observed about the MW was found to be low compared to predictions of cosmological simulations (the so-called "missing satellite problem;" Klypin et al. 1999). However, recent searches have revealed increased numbers of extremely faint dSphs (Walsh et al. 2009; Belokurov et al. 2006). When considered together with survey selection effects, the shortfall of local dSphs is substantially ameliorated (Koposov et al. 2009).

Secondly, among metal-poor stars $([\mathrm{Fe} / \mathrm{H}<-2.5 \mathrm{dex})$ the MDF and chemistry of the Galactic halo were seen to be very different from those exhibited by dSphs (Geisler et al. 2007). For example, the metal-poor tail of the MDF in dSphs was seen to be deficient compared to that of the halo (Helmi et al. 2006). However, subsequent studies of Starkenburg et al. (2010) do not uphold this deficiency. Schörck et al. (2009), Cohen \& Huang (2009), and Norris et al. (2008) conclude that the MDF of dSphs and the halo are in good agreement. Furthermore, evidence now shows that the extremely metal-poor stars in dSphs show similar $[\alpha / \mathrm{Fe}]$ to the halo (Frebel et al. 2010; Norris et al. 2010). It is therefore possible that present-day dSphs could have contributed to the metal-poor halo.

However, divergent chemistry remains at higher metallicity. For $[\mathrm{Fe} / \mathrm{H}]>-1$, the $[\alpha / \mathrm{Fe}]$ of the dSph sample is uniformly, and significantly, lower than that of the halo. Only Sgr, thought to be (in the form of its progenitor) the most massive of the local dwarf galaxies, has some stars with $[\alpha / \mathrm{Fe}]$ similar to the halo at intermediate metallicities. Signature of slow chemical enrichment, the $[\alpha / \mathrm{Fe}]$ of an average low-mass dSph implies that such systems cannot be responsible for a substantial fraction of the metal-poor halo.

In order to explain the halo chemistry, it is necessary to capture $[\mathrm{Fe} / \mathrm{H}] \gtrsim-2$ and $\alpha$-enhanced material. One mechanism to introduce such material is from a number of massive satellites that experienced rapid star formation and were then accreted rapidly, before the onset of SNIa contributions (Robertson et al. 2005; Font et al. 2006; De Lucia \& Helmi 2008). Lagos et al. (2009) make a clear distinction between "building block" satellites (i.e., those that are accreted) and surviving satellites. From cosmological simulations, they find that "building blocks" collapse and form stars earlier than surviving satellites that instead form stars in a quiescent manner. Cooper et al. (2010) find that the merger of less than five massive $\left(10^{8} M_{\odot}\right)$ "building blocks" at early times $(1<z<7)$ can account for the Galactic halo.

Our findings show that the chemistry of the stars contributed by Sgr to the Galactic halo in the trailing arm debris is significantly different from that of the halo at similar $[\mathrm{Fe} / \mathrm{H}]$. Therefore, Sgr is not a "building block." The debris are increasingly metal-poor further from the main body of Sgr and, so, in terms of the $[\mathrm{Fe} / \mathrm{H}]$ the material is increasingly "halolike." However, we see no signs of convergence between the $[\alpha / \mathrm{Fe}]$ ratio in stars of the trailing arm and stars of similar $[\mathrm{Fe} / \mathrm{H}]$ from the Galactic halo. Consequently, our findings show that the fraction of the intermediate-metal-rich halo population that can have been contributed by the dissolution of Sgr-like objects over the last several Gyr cannot be substantial.

\section{SUMMARY}

In this study, we have presented the abundance analysis of 11 M-giant stars in two regions along the trailing arm of the Sgr dwarf. The two populations, together with existing data from the literature, enable us to explore the metallicity and chemistry of stars deposited into the Milky Way halo from Sgr over the past $\sim 1.3$ Gyr. Within our limited sample of $\sim 2-3$ Gyr old stars, we find a significant gradient in metallicity along the debris stream. The metallicity gradient is $-(2.4 \pm 0.3) \times 10^{-3} \mathrm{dex} /$ degree $\left(-(9.4 \pm 1.1) \times 10^{-4} \mathrm{dex} / \mathrm{kpc}\right)$ decreasing away from the main body of Sgr. The $[\alpha / \mathrm{Fe}]$ ratio at a given metallicity is seen to be approximately constant along the extent of the trailing arm. The change in metallicity along the stream can be understood to be due to the tidal disruption of a Sgr progenitor that possessed a radial gradient in $[\mathrm{Fe} / \mathrm{H}]$. Such a gradient could have arisen due to a radial decrease in the star formation efficiency and/or a radial increase in the efficiency of galactic wind-driven metal loss. The physical mechanism that produced a radial $[\mathrm{Fe} / \mathrm{H}]$ gradient that persisted over a Hubble time did not, however, produce a detectable gradient in $[\alpha / \mathrm{Fe}]$.

There is no significant $[\alpha / \mathrm{Fe}]$ gradient along the trailing arm from our sample of M giants. The $[\alpha / \mathrm{Fe}]$ ratios of our targets are distinct from the halo. It can therefore be concluded that while some portion of the metal-poor halo might be contributed via the accretion of Sgr-like objects, the metal-rich component of the halo cannot feature a substantial contribution from such objects over the last several Gyr.

We thank Jorge Meléndez for providing the line list. This research has been supported in part by the Australian Research Council through Discovery Project grants DP0343962 and DP0878137. This is based on observations obtained at the Gemini Observatory for Program GS-2008B-Q-33, which 
is operated by the Association of Universities for Research in Astronomy, Inc., under a cooperative agreement with the NSF on behalf of the Gemini partnership: the National Science Foundation (United States), the Science and Technology Facilities Council (United Kingdom), the National Research Council (Canada), CONICYT (Chile), the Australian Research Council (Australia), Ministerio da Ciencia e Tecnologia (Brazil), and Ministerio de Ciencia, Tecnologia e Innovacion Productiva (Argentina).

Facilities: Gemini:South (Phoenix)

\section{REFERENCES}

Abadi, M. G., Navarro, J. F., \& Steinmetz, M. 2006, MNRAS, 365, 747 Asplund, M., Grevesse, N., \& Sauval, A. J. 2006, Nucl. Phys. A, 777, 1 Bell, E. F., et al. 2008, ApJ, 680, 295

Belokurov, V., et al. 2006, ApJ, 642, L137

Bessell, M. S., Castelli, F., \& Plez, B. 1998, A\&A, 333, 231

Bessell, M. S., Wood, P. R., \& Evans, T. L. 1983, MNRAS, 202, 59

Carollo, D., et al. 2007, Nature, 450, 1020

Chou, M., Cunha, K., Majewski, S. R., Smith, V. V., Patterson, R. J., MartinezDelgado, D., \& Geisler, D. 2010, ApJ, 708, 1290

Chou, M., et al. 2007, ApJ, 670, 346

Cohen, J. G., \& Huang, W. 2009, ApJ, 701, 1053

Cooper, A. P., et al. 2010, MNRAS, 406, 744

De Lucia, G., \& Helmi, A. 2008, MNRAS, 391, 14

Duffau, S., Zinn, R., Vivas, A. K., Carraro, G., Méndez, R. A., Winnick, R., \& Gallart, C. 2006, ApJ, 636, L97

Eggen, O. J., Lynden-Bell, D., \& Sandage, A. R. 1962, ApJ, 136, 748

Fellhauer, M., et al. 2006, ApJ, 651, 167

Font, A. S., Johnston, K. V., Bullock, J. S., \& Robertson, B. E. 2006, ApJ, 646, 886

Frebel, A., Simon, J. D., Geha, M., \& Willman, B. 2010, ApJ, 708, 560

Geisler, D., Wallerstein, G., Smith, V. V., \& Casetti-Dinescu, D. I. 2007, PASP, 119,939

Gilmore, G., \& Wyse, R. F. G. 1991, ApJ, 367, L55

Gustafsson, B., Edvardsson, B., Eriksson, K., Jørgensen, U. G., Nordlund, Å., \& Plez, B. 2008, A\&A, 486, 951

Harbeck, D., Grebel, E. K., Holtzman, J., Guhathakurta, P., \& Brandner, W. 2001, AJ, 122, 3092

Helmi, A. 2004, ApJ, 610, L97

Helmi, A., \& White, S. D. M. 2001, MNRAS, 323, 529

Helmi, A., et al. 2006, ApJ, 651, L121

Hidalgo, S. L., Aparicio, A., Martínez-Delgado, D., \& Gallart, C. 2009, ApJ, 705,704

Hill, V., Francois, P., Spite, M., \& Primas, F. 2000, A\&A, 364, L19

Hinkle, K. H., et al. 2003, Proc. SPIE, 4834, 353

Ibata, R., Lewis, G. F., Irwin, M., Totten, E., \& Quinn, T. 2001, ApJ, 551, 294

Ibata, R. A., Gilmore, G., \& Irwin, M. J. 1994, Nature, 370, 194

Jurić, M., et al. 2008, ApJ, 673, 864

Keller, S. C. 2010, PASA, 27, 45

Keller, S. C., Bessell, M. S., Cook, K. H., Geha, M., \& Syphers, D. 2002, AJ, 124, 2039

Keller, S. C., da Costa, G. S., \& Prior, S. L. 2009, MNRAS, 394, 1045

Keller, S. C., Murphy, S., Prior, S., da Costa, G., \& Schmidt, B. 2008, ApJ, 678, 851

Keller, S. C., \& Wood, P. R. 2006, ApJ, 642, 834

Klypin, A., Kravtsov, A. V., Valenzuela, O., \& Prada, F. 1999, ApJ, 522, 82

Koposov, S. E., Yoo, J., Rix, H., Weinberg, D. H., Macciò, A. V., \& Escudé, J. M. 2009, ApJ, 696, 2179

Lagos, C. D. P., Padilla, N. D., \& Cora, S. A. 2009, MNRAS, 397, L31

Lanfranchi, G. A., \& Matteucci, F. 2004, MNRAS, 351, 1338
Lanfranchi, G. A., Matteucci, F., \& Cescutti, G. 2006, A\&A, 453, 67

Law, D. R., Johnston, K. V., \& Majewski, S. R. 2005, ApJ, 619, 807

Law, D. R., Majewski, S. R., \& Johnston, K. V. 2009, ApJ, 703, L67

Layden, A. C., \& Sarajedini, A. 2000, AJ, 119, 1760

Lee, M. G., Yuk, I., Park, H. S., Harris, J., \& Zaritsky, D. 2009, ApJ, 703, 692

Majewski, S. R., Skrutskie, M. F., Weinberg, M. D., \& Ostheimer, J. C. 2003, ApJ, 599, 1082

Marigo, P., Girardi, L., Bressan, A., Groenewegen, M. A. T., Silva, L., \& Granato, G. L. 2008, A\&A, 482, 883

Martínez-Delgado, D., Gómez-Flechoso, M. Á., Aparicio, A., \& Carrera, R. 2004, ApJ, 601, 242

Martínez-Delgado, D., Peñarrubia, J., Jurić, M., Alfaro, E. J., \& Ivezić, Z. 2007, ApJ, 660, 1264

Meléndez, J., \& Barbuy, B. 1999, ApJS, 124, 527

Meléndez, J., Barbuy, B., Bica, E., Zoccali, M., Ortolani, S., Renzini, A., \& Hill, V. 2003, A\&A, 411, 417

Meléndez, J., Barbuy, B., \& Spite, F. 2001, ApJ, 556, 858

Meléndez, J., et al. 2008, A\&A, 484, L21

Monaco, L., Bellazzini, M., Bonifacio, P., Buzzoni, A., Ferraro, F. R., Marconi, G., Sbordone, L., \& Zaggia, S. 2007, A\&A, 464, 201

Monaco, L., Bellazzini, M., Bonifacio, P., Ferraro, F. R., Marconi, G., Pancino, E., Sbordone, L., \& Zaggia, S. 2005, A\&A, 441, 141

Muñoz, R. R., et al. 2005, ApJ, 631, L137

Newberg, H. J., Yanny, B., Cole, N., Beers, T. C., Re Fiorentin, P., Schneider, D. P., \& Wilhelm, R. 2007, ApJ, 668, 221

Norris, J. E., Gilmore, G., Wyse, R. F. G., Wilkinson, M. I., Belokurov, V., Evans, N. W., \& Zucker, D. B. 2008, ApJ, 689, L113

Norris, J. E., Yong, D., Gilmore, G., \& Wyse, R. F. G. 2010, ApJ, 711, 350

Pompéia, L., et al. 2008, A\&A, 480, 379

Prior, S. L., Da Costa, G. S., \& Keller, S. C. 2009, ApJ, 704, 1327

Prior, S. L., Da Costa, G. S., Keller, S. C., \& Murphy, S. J. 2009, ApJ, 691, 306

Robertson, B., Bullock, J. S., Font, A. S., Johnston, K. V., \& Hernquist, L. 2005, ApJ, 632, 872

Roškar, R., Debattista, V. P., Quinn, T. R., Stinson, G. S., \& Wadsley, J. 2008, ApJ, 684, L79

Sbordone, L., Bonifacio, P., Buonanno, R., Marconi, G., Monaco, L., \& Zaggia, S. 2007, A\&A, 465, 815

Schlegel, D. J., Finkbeiner, D. P., \& Davis, M. 1998, ApJ, 500, 525

Schörck, T., et al. 2009, A\&A, 507, 817

Searle, L., \& Zinn, R. 1978, ApJ, 225, 357

Shetrone, M., Venn, K. A., Tolstoy, E., Primas, F., Hill, V., \& Kaufer, A. 2003, AJ, 125,684

Siegel, M. H., et al. 2007, ApJ, 667, L57

Smith, G. H., Sneden, C., \& Kraft, R. P. 2002, AJ, 123, 1502

Sneden, C. 1973, ApJ, 184, 839

Spolaor, M., Proctor, R. N., Forbes, D. A., \& Couch, W. J. 2009, ApJ, 691, L138

Starkenburg, E., et al. 2009, ApJ, 698, 567

Starkenburg, E., et al. 2010, A\&A, 513, 34

Stinson, G. S., Dalcanton, J. J., Quinn, T., Gogarten, S. M., Kaufmann, T., \& Wadsley, J. 2009, MNRAS, 395, 1455

Tolstoy, E., Hill, V., \& Tosi, M. 2009, ARA\&A, 47, 371

Tolstoy, E., Venn, K. A., Shetrone, M., Primas, F., Hill, V., Kaufer, A., \& Szeifert, T. 2003, AJ, 125, 707

Unavane, M., Wyse, R. F. G., \& Gilmore, G. 1996, MNRAS, 278, 727

Venn, K. A., Irwin, M., Shetrone, M. D., Tout, C. A., Hill, V., \& Tolstoy, E. 2004, AJ, 128, 1177

Vivas, A. K., Jaffé, Y. L., Zinn, R., Winnick, R., Duffau, S., \& Mateu, C. 2008, AJ, 136, 1645

Vivas, A. K., et al. 2004, AJ, 127, 1158

Walsh, S., Willman, B., \& Jerjen, H. 2009, AJ, 137, 450

Yanny, B., et al. 2000, ApJ, 540, 825

Yanny, B., et al. 2009, ApJ, 700, 1282

Yong, D., Meléndez, J., Cunha, K., Karakas, A. I., Norris, J. E., \& Smith, V. V. 2008, ApJ, 689, 1020 